\title{
Impact of Moderate Intensity Aerobic Exercise on Irritable Bowel Syndrome in Post Cholecystectomy Patients
}

\author{
OMAR A.E. MOHAMED, M.Sc.*; MARWA M. EID, Ph.D.**; NESREEN A. ABD ELRASHID, Ph.D.** and \\ AHMED A.E. ALI, M.D.*** \\ The Department of Physical Therapist, Tamya General Hospital*, The Department of Surgery, \\ Faculty of Physical Therapy** and Plastic Surgery, Faculty of Medicine***, Cairo University, Egypt
}

\begin{abstract}
Background: Irritable bowel syndrome is a chronic functional disorder of the gastrointestinal system. Patients experience abdominal pain and altered bowel habit, with either predominantly diarrhea, constipation, or both, the risk to develop Irritable Bowel Syndrome (IBS) after cholecystectomy is 2.2 times higher than in general population.

Aim of Study: To evaluate the effect of moderate intensity aerobic exercise training (60\% MHR) (Treadmill Training) on Irritable Bowel Syndrome (IBS) symptom and Quality of Life in post cholecystectomy patients.

Patients and Methods: Thirty non-athletic post cholecystectomy patients complaining from Irritable Bowel Syndrome were recruited for this study; there age were ranged between 25 to 40 years, in the period between Oct. 2017 and Feb. 2019 from Kasr Al-Ainy Hospital. Patients who met the selection criteria were divided randomly into two equal groups, Group (A): They participated in 4 weeks of moderate intensity aerobic training program (60\% MHR Treadmill Training) (3 sessions per week) plus receiving medical treatment protocol, Group (B) (Medical Treatment Group) received medical care only.

Measurements: Irritable bowel syndrome was assessed by Irritable Bowel Syndrome Symptom Severity Score (IBSSSS) and Irritable Bowel Syndrome Quality of Life (IBSQOL) Instrument Assessment was carried out before treatment and after 4 weeks of treatment in both groups.

Results: The result showed that there was a significant decrease in post-treatment values of IBS-SSS and IBS-QOL in comparison to pre-treatment values at both groups with ( $p$ value $<0.001$ ). Post-treatment comparison between both groups showed significant difference in IBS-SSS and IBS-QoL variables, in favor of Group A (study group).

Conclusion: Moderate intensity aerobic exercise training (60\% MHR) for 4 weeks is effective in treatment of IBS post cholecystectomy through reducing IBS symptoms and improving quality of Life.
\end{abstract}

Key Words: -Irritable Bowel Syndrome (IBS) - Aerobic exercises - Post cholecystectomy.

Correspondence to: Dr. Omar A.E. Mohamed, The Department of Physical Therapist, Tamya General Hospital, Egypt

\section{Introduction}

IRRITABLE Bowel Syndrome (IBS) is a chronic functional disorder of the gastrointestinal system. Patients experience abdominal pain and altered bowel habit, with either predominantly diarrhea (IBS-D), constipation (IBS-C), or both (IBS-M). There is no definitive investigation as no biomarker has been found, so IBS is diagnosed clinically. The earliest reports describing IBS are from the turn of the 19 th and 20th century. At this time, diagnosis was only made by exclusion of malignant, inflammatory, or infectious disease after full investigation and extensive unsuccessful surgeries [1]

Irritable Bowel Syndrome is purported to be the most common reason for gastroenterologist visits and one of the top ten reasons for primary care appointments. Levy, Jones [2] determined that HMO subscribers with IBS accrued significantly higher healthcare costs ( $49 \%$ increase) and scheduled twice as many healthcare appointments as other HMO patients. In addition, people with functional gastrointestinal (GI) disorders appear to be increasingly turning to complementary and alternative treatments to find relief, with approximately $56 \%$ of one study's participants using alternative medicines or home remedies, while approximately $51 \%$ of that same study were taking nonprescription drugs [3]

Harvard Women's Health Watch (2009) identified IBS as the second leading cause of absenteeism from work; the common cold was first. In 2002, cases of IBS increased medical care costs by 10 billion U.S. dollars and were estimated to have contributed to 250 million lost days of work or school annually [4] 
Other estimates have run as high as 2 billion U.S. dollars in direct treatment costs and 20 billion dollars in indirect expenses [5].

Cholecystectomy is one of the most commonly performed abdominal surgical procedures, and in developed countries many are performed laparoscopically. As an example, 90 percent of cholecystectomies in the United States are performed laparoscopically. Laparoscopic cholecystectomy is considered the "gold standard" for the surgical treatment of gallstone disease. This procedure results in less postoperative pain, better cosmesis, shorter hospital stays and disability from work than open cholecystectomy. However, the overall serious complication rate in laparoscopic cholecystectomy remains higher than that seen in open cholecystectomy [6].

Laparoscopic cholecystectomy is becoming the choice of surgery for uncomplicated cholelithiasis because it had induced less tissue trauma response throughout the course of wound healing compared to open cholecystectomy [7].

The term Postcholecystectomy Syndrome (PCS) comprises a heterogeneous group of symptoms and findings in patients who have previously undergone cholecystectomy. Although rare, these patients may present with abdominal pain, jaundice or dyspeptic symptoms (IBS like symptoms). Many of these complaints can be attributed to complications including bile duct injury, biliary leak, biliary fistula and retained bile duct stones. Late sequelae include recurrent bile duct stones and bile duct strictures. With the number of cholecystectomies being performed increasing in the laparoscopic era the number of patients presenting with PCS is also likely to increase [8]

Chronic diarrhea is defined as an increase in stool frequency and/or volume that persists for longer than three to four weeks. Chronic symptoms generally do not suggest an infectious etiology, although patients may report that symptoms are preceded by gastrointestinal infection or food poisoning. The most common causes in clinical practice are inflammatory syndromes of the small bowel or colon (e.g., Crohn Disease [CD], celiac disease); functional bowel disorders (e.g/, Irritable Bowel Syndrome [IBS]); neoplasia; pancreatic insufficiency resulting in maldigestion; intestinal dysmotility; and small bowel malabsorption (e.g/, postgastrointestinal surgery) Bile Acid Malabsorption (BAM) is a common but frequently underrecognized cause of chronic diarrhea, with an estimated prevalence of $4 \%$ to $5 \%$ [9]
Urgency of defecation is excessively common postcholecystectomy, at least in women, and there is a tendency to more feelings of incomplete evacuation, suggesting that the rectum is more sensitive or irritable [10].

Epidemiological, clinical and basic research clearly demonstrated that regular exercise can prevent a variety of chronic diseases, such as cardiovascular diseases, diabetes, obesity, and enhance overall physical and psychological health from a young age. For example, physically, regular exercise in childhood improves strength and endurance, helps build healthy bones and muscles, helps control weight, improves blood pressure, reduces heart rate, and increases cholesterol levels [11].

Psychologically, regular exercise can reduce anxiety and stress as well as improve happiness, self-confidence, and boost self-esteem. The numerous health benefits gained from regular exercise are dependent on the type, intensity and volume of activity pursued by the individual [12]

So, the main aim of this study was to determine the effect of moderate intensity aerobic exercise training (60\% MHR) (treadmill training) on IBS symptom, associated pain and quality of life in post cholecystectomy patients.

\section{Material and Methods}

\section{A- Participants:}

A statistical power analysis suggested that sample size above 15 participants per group were required to achieve more than $80 \%$ power.

Thirty non-athletic post cholecystectomy patients, from both sexes, complaining from Irritable Bowel Syndrome were recruited for this study, there age was ranged between 25 to 40 . Patients were selected from Kasr Al-Ainy Hospital in the period between Oct. 2017 and Feb. 2019. Patients have been selected if they were medically stable and had a cholecystectomy operation 3 weeks ago [13]. All patients were examined by physician to exclude if they were had, cardiovascular or orthopedic problems, neurological or musculoskeletal disorders, mental disorder (it makes cooperation impossible), renal failure, myocardial infarction, severe asthma, diabetes, hypertensive, autoimmune disease or smokers subjects.

\section{They were assigned to two groups randomly:}

- Group A: This group included 15 non-athletic post cholecystectomy patients complaining from 
Irritable Bowel Syndrome. They participated in 4 weeks of moderate intensity aerobic training program (60\% MHR Treadmill Training) (3 sessions per week) plus receiving medical treatment protocol.

- Group B: This group included 15 non-athletic post cholecystectomy patients complaining from Irritable Bowel Syndrome. They received the medical treatment protocol only. Medical treatment outlines were Dietary treatment, Psychological treatment, and Pharmacological treatments outlines for IBS (Antidepressants, Fibers and laxatives for IBS-C, Antidiarrhoeal agents for IBS-D and Serotonin receptor agonists/ antagonists) [14]

The study has been approved by Ethical Committee in Faculty of Physical Therapy, Cairo University.

\section{B-Study design and randomization:}

The study was designed as a prospective randomized clinical trial in which patients were assigned randomly into two groups. Randomization was used to eliminate the researches' bias and was carried out by a blinded and an independent research assistant who opened sealed envelopes that contained a computer generated randomization card.

\section{C- Instrumentations:}

Standard weight scale, was used to measure weight and height and Body Mass Index (BMI) then calculated for each patient.

\section{1-Irritable Bowel Syndrome (IBS) Severity Score Procedures:}

The questionnaire has pain question, abdominal distension question, satisfaction with bowel habit and how much the IBS been affecting or interfering with patients life in general. The responses to all questions was measured on a ten points scale from (0) no distension to (10) very severe [14-16]

\section{2- Irritable Bowel Syndrome Quality ofLife (IBS- QOL) Instrument:}

The questionnaire consists of 32 questions asks about the effect of IBS and the psychological, social and daily living activates and the scale can be subdivided into 7 subscale namely Dysphoria section, interference with activity, body image, health worry, food avoidance, social reaction, sexual, and relationship [17-19].

Items $1,2,4,8-10,12,13,16,25-29$, and 34 use the following response scale ( $1=$ not at all, $2=$ slightly, $3=$ moderately, $4=$ quite a bit, and $5=\mathrm{ex}$ - tremely). Items $3,5-7,11,14,15,17-24$ and $30-$ 33 use the following response scale: ( $1=$ not at all, $2=$ slightly, $3=$ moderately, $4=$ quite a bit, and $5=\mathrm{a}$ great deal).

Subscale structure were, Dysphoria: Items 1, $6,7,9,10,13,16$, and 30 , interference with activity: Item $3,18,19,22,27,29$, and 31 , body image: Items 5, 21, 25, and 26, health worry: Items 4, 15, and 32 , food avoidance: Items 11, 23, and 28, social reaction: Items $2,14,17$, and 34 , sexual: Items 12 and 20, and relationship: Items 8, 24, and 33.

\section{D- Treatment procedures:}

Both groups (A and B) received the same medical treatment program throughout the treatment period. In addition to the medical treatment, Group A received moderate intensity aerobic exercises as following:

Using a program of exercise with intensity of maximum heart rate $60 \%$ for Group $\mathrm{A}$ and frequency of 3 times/week for 4 weeks. The session consists of 5 minutes warm-up performed on the treadmill at low load followed by training period for 30 minutes and ended by 5 minutes cool down [20] as following:

1- Warming up exercise: The aerobic training program included a 5-min warm-up consisting of fast walking, slow running and stretching [21].

2- Exercise phase: After warming up, continuous running was performed started by walking $~ 2.0$ $\mathrm{mph}$ increased gradually every $2 \mathrm{~min}$ to reach intensity of $60 \%$ of the maximum heart rate of the participant. The running period was $15 \mathrm{~min}$ for the first session and every two sessions 2 mins were added to the running period in a stepwise manner until the running period reached 30min [22]

3- Cool down exercise: At the end of each session there was a cool-down period consisting of slow running and stretching for $5 \mathrm{~min}$ [21]

\section{E- Statistical analysis:}

Data were collected before initiation of the treatment (pre-treatment), and after 4 weeks of treatment (post-treatment). Statistical analysis was conducted using SPSS for Windows, Version 25 (SPSS, Inc., Chicago, IL).

The collected data were analyzed statistically by the descriptive statistics, based on the raw data, where mean and standard deviation of age, height, 
weight, BMI, IBS-SSS and IBS-QoL variables were calculated for all patients. Inferential statistical analysis and comparison of IBS-SSS and IBS-QoL were made by paired sample $t$-tests for within group comparison, between pre and post-treatment results, while independent sample $t$-test was used for comparison between groups at pre and posttreatment periods. Independent $t$-test was used for comparison between groups age (years), weight $(\mathrm{Kg})$, height $(\mathrm{cm})$ and BMI $\left(\mathrm{kg} / \mathrm{m}^{2}\right)$ variables. The level of significance was set at the 0.05 level.

\section{Results}

\section{F- Baseline and demographic data:}

There were no statistically significant differences $(p>0.05)$ between subjects in both groups concerning age, body mass, height, BMI, IBS-SSS or IBS-QoL (Table 1).

\section{1-IBS-SSS results and statistical analysis:}

As presented in (Table 2), within group's comparison the mean values of body IBS-SSS in the "pre" and "post" tests revealed significant reduction at post-treatment in comparison to pre-treatment in both Groups A and B with $p$-value $<0.001$. Between groups comparison for mean values of IBS-SSS showed significant difference posttreatment with $(p<0.0001)$ between both groups with favor of Group A.

\section{2-IBS-QoL results and statistical analysis:}

Regarding IBS-QoL (Table 2) showed significant reduction of IBS-QoL score at post-treatment in comparison to pre-treatment in both Groups A and $\mathrm{B}$ with $p$-value $<0.001$. Between groups comparison for mean values of IBS-QoL showed significant difference post-treatment with $(p<0.0001)$ between both groups with favor of Group A.

Table (1): General characteristics of the two studied groups (A and B groups).

\begin{tabular}{|c|c|c|c|c|c|}
\hline \multirow{2}{*}{ Items } & \multirow{2}{*}{$\begin{array}{c}\text { Group A } \\
\text { Mean } \pm \text { SD }\end{array}$} & \multirow{2}{*}{$\begin{array}{c}\text { Group B } \\
\text { Mean } \pm \text { SD }\end{array}$} & \multicolumn{2}{|c|}{ Comparison } & \multirow{2}{*}{ S } \\
\hline & & & $p$-value & $t$-value & \\
\hline Age (yrs) & $31.6 \pm 4.579$ & $32.13 \pm 5.29$ & -0.295 & 0.77 & NS \\
\hline Weight (Kg) & $71.87 \pm 11.426$ & $77.6 \pm 8.959$ & -0.441 & 0.662 & NS \\
\hline Height $(\mathrm{cm})$ & $169.67 \pm 9.854$ & $171.13 \pm 8.28$ & -1.529 & 0.137 & NS \\
\hline $\mathrm{BMI}\left(\mathrm{Kg} / \mathrm{m}^{2}\right)$ & $24.93 \pm 3.173$ & $26.47 \pm 2.232$ & -1.531 & 0.137 & NS \\
\hline IBS-SSS & $382.667 \pm 31.045$ & $376.667 \pm 33.947$ & 0.505 & 0.617 & NS \\
\hline IBS-QoL & $139.533 \pm 11.025$ & $144.8 \pm 7.523$ & -1.528 & 0.138 & NS \\
\hline
\end{tabular}

Table (2): Statistics of IBS-SSS and IBS-QoL and statistical analysis in post-treatment for both groups.

\begin{tabular}{lllll}
\hline & $\begin{array}{c}\text { Group A } \\
\text { Mean } \pm \text { SD }\end{array}$ & $\begin{array}{c}\text { Group B } \\
\text { Mean } \pm \text { SD }\end{array}$ & MD & $\begin{array}{c}p \text { - } \\
\text { value }\end{array}$ \\
\hline IBS-SSS & 11213.732 & 18217.809 & -70 & $<0.001^{*}$ \\
IBS-QOL & 60.3336 .883 & 94.2675 .257 & -33.933 & $<0.001^{*}$ \\
\hline
\end{tabular}

$\begin{array}{ll}* & : \text { Significant level is set at alpha level }<0.05 . \\ \text { SD } & : \text { Standard Deviation. } \\ \text { MD } & : \text { Mean Difference. } \\ p \text {-value : Probability value. }\end{array}$

\section{Discussion}

This study was conducted to investigate and compare between the effect of moderate intensity aerobic exercise plus medical treatment versus medical treatment only in patients with irritable bowel syndrome post cholecystectomy.

\section{The results of the study showed that:}

The pre-treatment results of the present study revealed no significant difference between the mean values of IBS-SSS and IBS-QoL between both groups Group A (study group) and Group B (control group). The post-treatment results of this study by IBS-SSS showed that there was significant difference between both groups Group A (study group) (with percentage of improvement $70.7 \%$ ) and Group B (control group) (with percentage of improvement $51.7 \%$ ) with ( $p$-value $<0.001)$, in similarity with the post-treatment results of this study by IBS-QoL which showed that there was also significant difference between both groups [Group A (study group) (with percentage of improvement 56.8\%)] and Group B (control group) (with percentage of improvement $34.9 \%$ ) with ( $p$ value $<0.001$ ), in favor of Group A (study group).

Regarding the effect of the moderate intensity aerobic exercises on irritable bowel syndrome, our results came in consistnce with Maleki, Tartibian [23] who examined the effect of moderate aerobic exercise, versus non exercise control on IBS severity symptoms evaluated after 24 weeks, in 109 
female volunteers sedentary IBS patients (aged $18-41 \mathrm{yrs}$ ) who fulfilled Rome III criteria. The resultes reported low-to-moderate intensity exercise training program attenuates symptoms in IBS.

Our results came in agreement with Johannesson, Simrén [24] who demonstrated a significant improvement in the IBS-SSS score between the physical activity group and the control group (51 (-130 and 49) Vs. -5 (-101 and 118), $p=0.003)$. The proportion of patients with increased IBS symptom severity during his study was significantly larger in the control group than in the physical activity group. Also, Morlin, Simren [25] had similar results of our study when they evaluated the effect of increased physical activity on fatigue, depression, anxiety and GI-symptoms in 94 (20M) patients with IBS and Rome II criteria. The subjects were randomised to a control group (35F/12M) or a training group $(39 \mathrm{~F} / 8 \mathrm{M})$ : The IBS-score in the training group was $292(133-371)$ at start and 230 (104-370) after 12 weeks, $p<0.001$. In the controls it was 278 (148-378) at start and 264 (133-386) at 12 weeks, $p=N S$. So Morlin, Simren [25] study concluded that increased physical activity is associated with symptom improvement in IBS but does not reduce fatigue or depression and anxiety in patients with IBS. Also, our results came in agreement with De Schryver, Keulemans [26] who studied the influence of regular physical activity on colonic transit time and defecation in middle-aged inactive patients suffering from chronic idiopathic constipation, by application on forty-three subjects ( $>45$ years) were randomly divided into Group $\mathrm{A}(\mathrm{n}=18$, $16 \mathrm{~F}, 2 \mathrm{M})$ and Group B $(\mathrm{n}=25,20 \mathrm{~F}, 5 \mathrm{M})$. Group A subjects maintained their normal lifestyle during 12 weeks, followed by a 12 -week PA programme. Group B performed a 12-week PA programme after randomization. PA comprised 30min of brisk walking and a daily 11-min home-based programme, in Group B a significant reduction in 3 out of 4 of the Rome I criteria for constipation was observed, i.e. percentage of incomplete defecations, percentage of defecations requiring straining and percentage of hard stools $(p<0.05)$. As a consequence, the number of fulfilled Rome criteria for constipation decreased ( 2.7 to $1.7 ; p<0.05)$. Furthermore, the rectosigmoid and total colonic transit time decreased (17.5 to $9.6 \mathrm{~h}$ and 79.2 to $58.4 \mathrm{~h}$, respectively; $p<0.05)$. After PA the number of fulfilled Rome criteria also decreased in Group A (2.6 to 1.7; $p<0.05)$. Which concluded that regular physical activity improved both the defecation pattern and rectosigmoid or total colonic transit time in middleaged inactive subjects with symptoms of chronic constipation. Our results are similar to the findings of Villoria, Serra [27] who evaluated the putative beneficial effects of physical activity in patients with abdominal bloating, application was on eight patients complaining of bloating, seven with irritable bowel syndrome, and one with functional bloating, a significant proportion of the gas infused was retained in the gut $(45 \pm 9 \%, p<0.01$ vsbasal), but retention was significantly lower during exercise $(24 \pm 7 \%, p<0.05$ vs rest). Gas retention during rest was associated with significant abdominal symptoms (3.6 score; $p<0.01 \mathrm{Vs}$. basal), and symptoms also improved during exercise ( 2.8 score, $p$ $<0.05$ Vs. rest). Which confirms that mild exercise enhances intestinal clearance and prevents retention of intestinal gas loads. Our results also came in accordance with another study for Lustyk, Jarrett [28] who tested the effect of physical activity in women with irritable bowel syndrome, this study was done on 89 women women with irritable bowel syndrome were significantly less likely to be active (48\%) than control women $(71 \%)\left(\chi^{2}=3.4, p=.05\right)$. Within the irritable bowel syndrome group, active women were less likely to report a feeling of incomplete evacuation following a bowel movement than inactive women $(p<.04)$, yet active women did not have less severe recalled psychological or somatic symptoms than inactive women. Active women with irritable bowel syndrome reported less severe daily somatic symptoms, which were accounted for by a lower level of fatigue ( $p$ $=.003$ ), but not daily GI or psychological symptoms. Regarding our results that aerobic exercise is more effective than medical treatment alone, results of this study supports the results of Feng, Bian [29] who compare between the efficacy of Baduanjin exercise on the constipation-predominant Irritable Bowel Syndrome of the elderly plus Tegasero medical treatment versus receiving Tegasero medical treatment only, by application on 60 elderly patients were randomly divided into experiment group and control group, with 30 patients each. The experiment group did Baduanjin exercise and received Tegasero and the control group received Tegasero only. The treatment lasted 12 weeks. Result: The treatment was effective for both the groups. But the efficacy was better for the experiment group in regard of total curative effect, symptom improvement and stool characters $(p=0.05)$ which indicated that: Combination of Baduanjin exercise and Tegasero may treat constipationpredominant irritable bowel syndrome of the elderly more effectively. On the other hand, our study is partially disagreed with results of Daley, Grimmett [30] who studied the effects of an exercise intervention upon quality of life and irritable bowel symptoms. Outcomes included irritable bowel specific quality of life, symptoms (total symptoms, 
constipation, diarrhoea and pain). Analyses revealed no differences in quality life scores between groups at 12-week follow-up. The exercise group reported significantly improved symptoms of constipation (mean difference $=10.9,95 \% \mathrm{CI}=-20.1,-1.6)$ compared to usual care at follow-up. The non-significant difference between both groups total quality of life score may be due to that the patient asked to do $30 \mathrm{~min}$ moderate exercises, five days per week, at home with no supervision, which may results in not achieving the sited exercise goal for all patients, while in our study subjects do exercises in the clinic under our supervision, for $45 \mathrm{~min}, 3$ times per week.

The effect of aerobic exercise on IBS could be explained by the following mechanisms:

Decreased gastrointestinal blood flow, increased gastrointestinal motility, increased mechanical bouncing, and altered neuroendocrine modulation, decreased biliary cholesterol secretion, and enhanced gall bladder and colonic motility are postulated [31]. Exercise intervention favorably attenuated inflammation as indicated by plasma cytokines (IL-1 3 , IL-6, IL-8, IL-10 and TNF-a), adenosine deaminase, oxidative stress (XO, MDA and NO) and enhanced antioxidants (SOD, CAT and GSH-Px). So, symptom improvement was associated with a reversal of the ratio of anti-to pro-inflammatory cytokines as well as facilitating blood redox homeostasis, suggesting an immuneand redox modulating function for exercise training when the changes in blood oxidative stress status, inflammatory biomarkers [23]

\section{Conclusions:}

The results of current study showed that moderate intensity aerobic exercise training $(60 \%$ MHR) for 4 weeks is effective in treatment of IBS post cholecystectomy through reducing IBS symptoms and improving quality of Life.

\section{Recommendations:}

The results of the study had indicated need to consider the following recommendations: Future studies are recommended to include patients with different age group. Future studies are recommended using different aerobic exercises parameters. Future studies are recommended using different types of exercises (underwater exercises, strengthening or endurance exercises). Further follow-up studies of various forms of assessment for those patients are recommended. Future studies are recommended using aerobic exercises on IBS post different types of operations. Further researches should be extended to a large number of patients.

\section{Acknowledgement:}

Author would like to thank Dr. Marwa M. Eid and Dr. Nesreen Afify for their help and support, and Dr. Ahmed Ali for his guidance.

\section{References}

1- CANAVAN C., J. WEST and T.R. CARD: The epidemiology of irritable bowel syndrome. Clinical Epidemiology, 6: p. 71-80, 2014.

2- LEVY R.L., et al.: Irritable bowel syndrome in twins: Heredity and social learning both contribute to etiology. Gastroenterology, 121 (4): p. 799-804, 2001.

3- NYROP K., et al.: Costs of health care for irritable bowel syndrome, chronic constipation, functional diarrhoea and functional abdominal pain, 26 (2): p. 237-48, 2007.

4- BLANCHARD E.B., L.J.J.O.C. SCHARFF and c. Psychology, psychosocial aspects of assessment and treatment of irritable bowel syndrome in adults and recurrent abdominal pain in children, 70 (3): p. 725, 2002.

5- SANDLER R.S., et al.: The burden of selected digestive diseases in the United States, 122 (5): p. 1500-11, 2002.

6- JOHANSSON M., et al.: Randomized clinical trial of open versus laparoscopic cholecystectomy in the treatment of acute cholecystitis. British Journal of Surgery, 92 (1): p. 44-9, 2005 .

7- LUO K., et al.: Operative stress response and energy metabolism after laparoscopic cholecystectomy compared to open surgery. World Journal of Gastroenterology, 9 (4): p. 847-50, 2003.

8- JAUNOO S., S. MOHANDAS and L. ALMOND: Postcholecystectomy syndrome (PCS). International Journal of Surgery, 8 (1): p. 15-7, 2010.

9- BARKUN A., et al.: Bile acid malabsorption in chronic diarrhea: Pathophysiology and treatment. Canadian Journal of Gastroenterology and Hepatology, 27 (11): p. 653-9, 2013.

10- HEATON K., D. PARKER and H. CRIPPS: Bowel function and irritable bowel symptoms after hysterectomy and cholecystectomy-A population based study. Gut, 34 (8): p. 1108-11, 1993.

11- YETTER G.: Exercise-based school obesity prevention programs: An overview. Psychology in the Schools, 46 (8): p. 739-47, 2009.

12-NETZ Y., et al.: Physical activity and psychological wellbeing in advanced age: A meta-analysis of intervention studies. Psychology and aging, 20 (2): p. 272, 2005.

13- TAMHANKAR A.P., et al.: Post-operative symptoms, after-care, and return to routine activity after laparoscopic cholecystectomy. JSLS: Journal of the Society of Laparoendoscopic Surgeons, 14 (4): p. 484, 2010.

14- SPILLER R., et al.: Guidelines on the irritable bowel syndrome: Mechanisms and practical management. J. Gut., 56 (12): p. 1770-98, 2007.

15- FRANCIS C., J. MORRIS and P. WHORWELL: The irritable bowel severity scoring system: A simple method of monitoring irritable bowel syndrome and its progress. Alimentary pharmacology \& therapeutics, 11 (2): p. 395402, 1997. 
16- DROSSMAN D.A., et al.: Severity in irritable bowel syndrome: A Rome foundation working Team report. The American Journal of Gastroenterology, 106 (10): p. 1749, 2011.

17- DROSSMAN D.A., et al.: Further validation of the IBSQOL: A disease-specific quality-of-life questionnaire. The American Journal of Gastroenterology, 95 (4): p. 999-1007, 2000.

18- PARÉ P., et al.: Health-related quality of life, work productivity, and health care resource utilization of subjects with irritable bowel syndrome: Baseline results from LOGIC (Longitudinal Outcomes Study of Gastrointestinal Symptoms in Canada), A Naturalistic Study, 28 (10): p. 1726-35, 2006.

19- ANDRAE D.A., et al.: Evaluation of the Irritable Bowel Syndrome Quality of Life (IBS-QOL) questionnaire in diarrheal-predominant irritable bowel syndrome patients. Health and quality of life outcomes, 11 (1): p. 208, 2013.

20- TENZIN K., N. BEGUM and S. FERDOUSI: Effect of moderate physical exercise on autonomic Balance in Irritable Bowel Syndrome. Journal of Bangladesh Society of Physiologist, 10 (1): p. 6-10, 2015.

21- HILL E., et al.: Exercise and circulating cortisol levels: The intensity threshold effect. Journal of Endocrinological Investigation, 31 (7): p. 587-91, 2008.

22- KETEYIAN S.J.: Graded exercise testing and exercise prescription., In clinical exercise physiology, J.K. Ehrman, et al., Editors, Human Kinetics: Champaign, Illinoi. p. 61-88, 2013.

23- MALEKI B.H., et al.: Low-to-moderate intensity aerobic exercise training modulates irritable bowel syndrome through antioxidative and inflammatory mechanisms in women: Results of a randomized controlled trial. Cytokine, 102: p. 18-25, 2018.
24- JOHANNESSON E., et al.: Physical activity improves symptoms in irritable bowel syndrome: A randomized controlled trial. The American Journal of Gastroenterology, 106 (5): p. 915-22, 2011.

25- MORLIN E., et al.: M1775 increased physical activity improves GI-symptoms but not fatigue and depression in the irritable bowel syndrome (IBS). Gastroenterology, 134 (4): p. A-416, 2008.

26- De SCHRYVER A.M., et al.: Effects of regular physical activity on defecation pattern in middle-aged patients complaining of chronic constipation. Scandinavian Journal of Gastroenterology, 40 (4): p. 422-9, 2005.

27- VILLORIA A., et al.: Physical activity and intestinal gas clearance in patients with bloating. The American Journal of Gastroenterology, 101 (11): p. 2552, 2006.

28- LUSTYK M., et al.: Does a physically active lifestyle improve symptoms in women with irritable bowel syndrome? Gastroenterology nursing: The Official Journal of the Society of Gastroenterology Nurses and Associates, 24 (3): p. 129-37, 2001.

29- FENG Y., et al.: Observation of the efficacy of Baduanjin exercise on the constipation-predominant irritable bowel syndrome of the elderly. Sport Sci. Res., 31 (2): p. 8998, 2010.

30- DALEY A., et al.: The effects of exercise upon symptoms and quality of life in patients diagnosed with irritable bowel syndrome: A randomised controlled trial. International Journal of Sports Medicine, 29 (09): p. 778-82, 2008.

31- PETERS H., et al.: Potential benefits and hazards of physical activity and exercise on the gastrointestinal tract. Gut., 48 (3): p. 435-9, 2001. 


\section{تآثير التمارين الهوائية المتوسطة الشدة على متلاز مة القولون العصبى الهبى

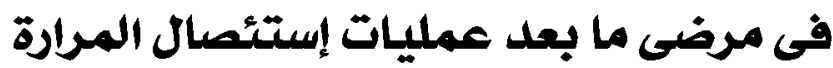

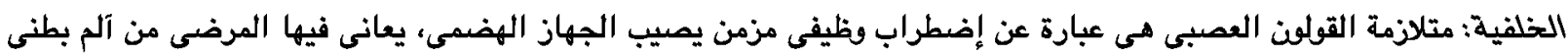

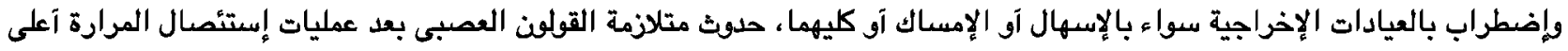


الهدف من البحث: آجريت هذه الدراسة بهدف معرفة فعالية التمارين الهوائية المتوسطة على متلازمة القولمن العصبى فى مرضى ما بعد عمليات إستئسال المرارة.

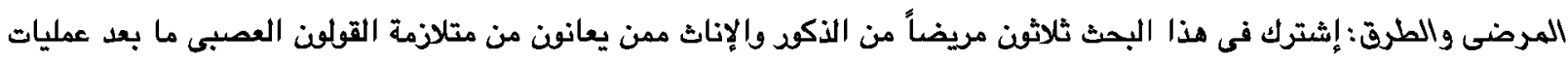

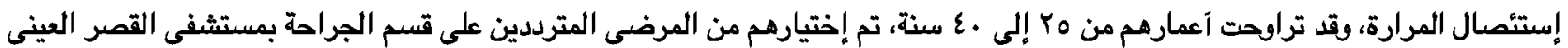

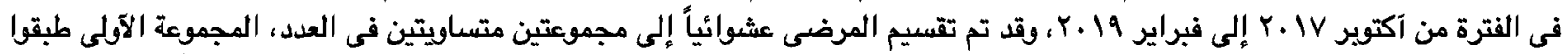



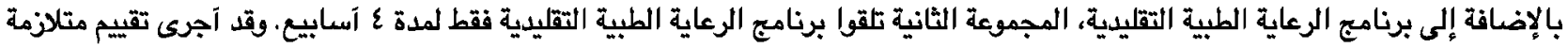
القولون العصبى بمقياس درجة شدة الآعراض ومقياس جودة العياة العياة المتلازمة، تم التقييم قبل العلاج وبعد ع آسابيع من العلاج في كلا المجموعتين لجميع المرضى فى المجموعتين.

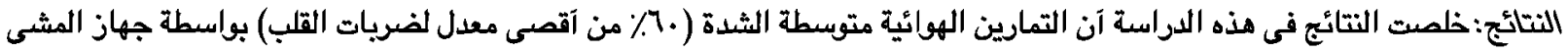


إستئصال المرارة. 